\title{
La nucléocapside du virus humain HIV-1
}

Le colloque "Basic Features of the Nucleocapsid of the Human Immunodeficiency Virus Type 1 ", organisé à Lyon en avril 1993 par Jean-Luc Darlix et Bernard-Pierre Roques, sous l'égide de l'Ecole normale supérieure de Lyon, avait pour but de faire le point de nos connaissances sur la structure intime et les fonctions de la nucléocapside du virus humain HIV-1, l'agent causal du SIDA.

HIV-1 est un virus globulaire enveloppé, ayant un diamètre d'environ 110-120 nm. Le noyau viral, encore appelé capside, renferme la nucléocapside, qui est l'élément le plus stable du virus. Comme dans les rétrovirus aviaires et murins fort étudiés depuis vingt ans, la nucléocapside du HIV-1 est constituée de l'ARN génomique diploïde sur lequel sont fixées environ 1500 molécules d'une petite protéine basique, dénommée $\mathrm{NCp} 7$, qui possède deux doigts à zinc de type $\mathrm{CX}_{2} \mathrm{CX}_{4} \mathrm{HX}_{4} \mathrm{C}^{*}$ (figure 1). Cependant, à l'intérieur de la nucléocapside du HIV-1, les interactions entre les molécules de $\mathrm{NCp} 7$ et le génome dimérique, ainsi que le mode d'association entre les deux molécules d'ARN génomique au sein du dimère, restent mal connus. Il en est de même de la formation et des fonctions de la nucléocapside du HIV-1, et de celle des rétrovirus en général. Cette nucléocapside contient également des enzymes comme la transcriptase inverse, l'intégrase et la protéase (environ 50 molécules de chaque) ainsi que des molécules d'ARNt

$\overline{-C: C y s ; H}$ : His ; X: n'importe quel acide

dont l'ARNt ${ }^{\text {Lys,3 }}$ qui est l'amorce réplicative du HIV-1 [1].

Par ailleurs, et cela représente une remarquable condensation d'informations, les 500 premiers nucléotides du génome ARN du HIV-1 (appelés leader), domaine sur lequel se fixe la NCp7, sont impliqués : (1) dans l'expression virale : TAR au niveau de la transcription, signal d'épissage en 5', signal d'initiation de traduction ; (2) dans la formation du virus : phénomène unique de dimérisation du génome viral, encapsidation du génome ARN dimérique ; (3) dans sa réplication: hybridation de l'amorce réplicative $\mathrm{ARNt}^{\mathrm{Lyz}, 3}$ à son site de liaison PBS (polymerase binding site) et initiation de la transcription inverse, puis intégration après transcription inverse de l'ARN en ADN double brin.

L'étude de la $\mathrm{NCp} 7$ et de son rôle au niveau des multiples fonctions codées par le leader ARN (les 500 premiers nucléotides) a conduit à la mise en place de plusieurs collaborations depuis quatre ans. En effet, la taille de la NCp7, 72 acides aminés, est compatible avec sa synthèse ainsi que celle de mutants par voie chimique, et avec l'analyse fine par résonance magnétique nucléaire ( $\mathrm{RMN}$ ) de sa structure et de ses interactions avec l'ARN viral et l'amorce réplicative $A R t^{\text {Lys,3. }}$. De surcroît, les relations structure/fonctions de la protéine $\mathrm{NCp} 7$ sont faciles à étudier in vitro : reconnaissance de l'ARN viral et du

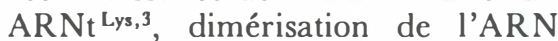
viral et initiation de la transcription inverse, et peuvent être transposées in vivo après mutagenèse dirigée et analyse des virus mutants et de leur pouvoir infectant.

Le dialogue ainsi établi entre la virologie, la génétique moléculaire, la biochimie et la biophysique a porté ses premiers fruits, et le colloque de Lyon a montré que nous commençons à comprendre le rôle des interactions entre la NCp7, le génome ARN et l'amorce ARNt ${ }^{\text {Lys, } 3}$ dans la morphogenèse, la structure et la réplication de l'agent infectieux, le HIV-1.

Il est à noter que l'exemple des travaux sur le HIV-1 peut aussi s'appliquer à l'étude d'autres rétrovirus comme les virus murin MuLV (murine leukaemia virus), aviaire ALV (avian leukosis virus) et simien SIV (simian immunodeficiency virus). Ces rétrovirus sont aussi d'excellents modèles d'étude pour analyser les effets de mutations dans la protéine $\mathrm{NC}$ et le leader 5' du génome ARN chez l'animal [2-5].

Les relations structure/fonctions du complexe NCp7 : ARN viral au cours du cycle réplicatif du HIV-1 La figure 1 présente très brièvement la structure génétique du provirus HIV-1 ainsi que la protéine NCp7, et la figure 2 résume les données récentes qui ont été largement discutées au cours de ce colloque. Certaines données, non encore publiées, seront brièvement présentées dans les commentaires qui suivent.

Une cellule infectée produit des virus, encore appelés virions, par un processus de bourgeonnement (figure 2). Ces bourgeons viraux résultent de multiples interactions qui interviennent au 
niveau de, et sous la membrane plasmique, entre des précurseurs polyprotéiques du virus, Gag et Gag-Pol, ancrés dans la membrane grâce à un résidu myristate en $\mathrm{N}$-terminal. Les interactions entre $\mathrm{Gag}$ et les protéines d'enveloppe, SUgpl20 et TMgp41, interviennent également dans la constitution du virus (toutes ces interactions ne sont pas représentées dans ce schéma).

L'encapsidation spécifique du génome viral doit aussi intervenir à ce stade pour produire un virus infectieux. Les données génétiques indiquent que la protéine $\mathrm{NCp} 7$ est directement impliquée dans le choix de l'ARN viral non épissé ("pré-génome ") et dans son encapsidation. Ces deux étapes seraient guidées par des structures uniques, présentes dans le leader de l'ARN viral, qui ressembleraient à une conformation en quadruple hélice (figure 3) [6-8]. En effet, la protéine $\mathrm{NCp} 7$ reconnaît une séquence riche en résidus $\mathrm{T}$ et $\mathrm{A}$ présente dans le signal d'encapsidation/dimérisation spécifique du génome viral (figure 3).

Les processus de bourgeonnement et d'encapsidation suscitent de nombreuses questions, en particulier : la protéine $\mathrm{NCp} 7$ agit-elle à l'état immature (précurseur Gag, voir figure 1) ou mûr ? l'ARN viral est-il encapsidé à l'état de monomère ou en tant que dimère ?

Il n'y a pas actuellement de réponse simple à la première question. En effet, l'ensemble des produits de maturation de Gag par la protéase (MApl7, CAp24, NCp7 et p6), ainsi que le précurseur Gag, sont présents dans les cellules infectées. Les virus dépourvus de protéase virale ne sont pas infectieux, de même que ceux produits par des cellules infectées cultivées en présence d'inhibiteurs de

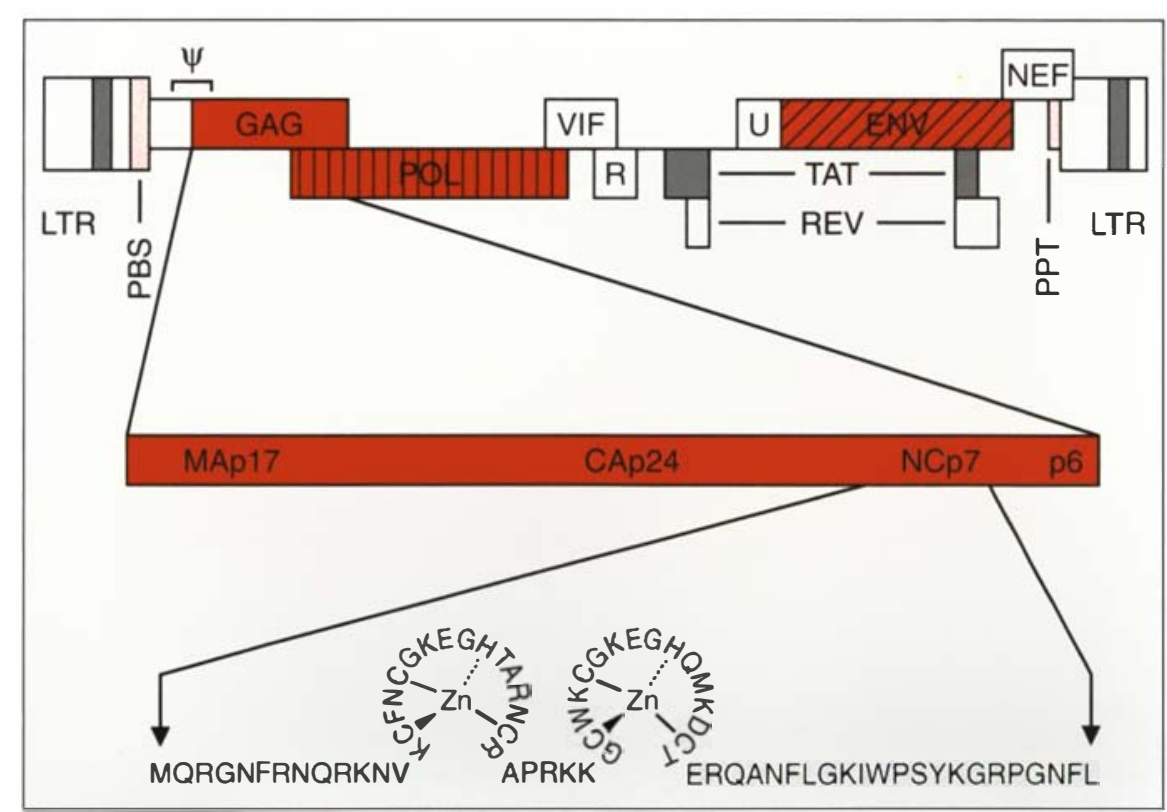

Figure 1. Représentation schématique de la structure génétique du provirus HIV-1. Les LTR contiennent les signaux d'initiation et de terminaison de la transcription du provirus, et leurs extrémités $5^{\prime}$ et $3^{\prime}$ sont nécessaires à l'intégration de l'ADN proviral dans I'ADN de la cellule hôte. Le PBS (primer binding site) est le site d'hybridation de l'ARNt'lys,3 et donc le site d'initiation de la transcription inverse (ADN de sens -) alors que la PPT (polypurine tract) est le site d'initiation de la synthèse de l'ADN viral de sens + . La séquence $\Psi$ correspond au signal de dimérisation : encapsidation du génome ARN. Les bornes des cistrons Gag et Pol sont indiquées, et la séquence de la NCp7 est détaillée avec les doigts à zinc dans des pseudo-cercles. Le cistron Pol code pour les enzymes du virus, qui sont, dans l'ordre: la protéase, la transcriptase inverse et l'intégrase (se reporter à [1] pour plus de détails). protéase. D'autre part, des mutations empêchant la maturation de la protéine NCp7 du précurseur Gag bloquent la production de particules virales (V. Housset). Ces deux résultats suggèrent un rôle préférentiel pour la NCp7 mûre. Cependant, une surproduction de la protéase virale conduit à une maturation très rapide de Gag dans les cellules infectées et à une très forte réduction de la quantité de virions produits. Cela pourrait provenir de l'inactivation des protéines Gag, et en particulier de la NCp7, par un excès de protéase (Wondrack, de Rocquigny). L'ensemble de ces résultats laisse supposer que l'assemblage des précurseurs Gag et Gag-Pol se fait de concert avec leur maturation (stades 1 et 2 de la figure 2).

La réponse à la deuxième question est plus simple. En effet, le génome viral est très probablement encapsidé sous forme dimérique dans les virions infectieux comme l'indiquent les arguments suivants : (1) de l'ARN génomique dimérique est observé dans les cellules infectées [4] ; (2) des virus ayant une ou plusieurs mutations dans la NCp7 sont fortement atténués et possèdent de l'ARN en faible quantité, mais encore sous forme dimérique. Enfin, des données de génétique moléculaire et de microscopie électronique renforcent l'idée selon laquelle la formation des virions est probablement un processus concerté où s'enchaînent et se coordonnent des interactions protéines:lipides, protéines:protéines, protéines:ARN, et ARN:ARN. Les interactions protéines:protéines, c'està-dire Gag:Gag et Gag:Gag-Pol, impliqueraient plusieurs domaines distincts de Gag (MA, CA et Nterminal de NCp7) et de Pol $[9,10]$. Une question importante, demeurée sans réponse, concerne la manière dont s'organise l'assemblage : autour d'une pré-nucléocapside comme site de nucléation, ou par incorporation de la nucléocapside à une précapside ? Une étape supplémentaire, correspondant à l'hybridation de l'amorce réplicative $\mathrm{ARNt}^{\text {Lys, } 3}$ au site PBS (étape 3, figure 2), est nécessaire à la formation de virus infectieux. Elle semble mettre en jeu des interactions entre l'ARN viral, 
l'AR ${ }^{\text {Lys,3 } 3}$ et la protéine $\mathrm{NCp} 7$. Cette étape pose de nombreuses questions parmi lesquelles: la mise en place de l'ARNt sur son site de liaison se fait-elle lors de la formation du virus ou plus tard? Le précurseur Gag peut-il induire cette hybridation? Des expériences préliminaires indiquent que Gag serait incapable d'hybrider ARNt ${ }^{\mathrm{Lys}, 3}$ au PBS, et que cette mise en place serait assez tardive, bien qu'un début de transcription inverse du génome ait été observé dans les virions.

L'entrée du virus dans la cellule, c'est-à-dire l'infection virale, met aussi en jeu des systèmes de reconnaissance entre macromolécules, SUgpl20 de l'enveloppe virale et CD4 de la cellule cible, TMgp41 et bicouche lipidique, qui conduisent à l'entrée de la capside dans le cytoplasme de la cellule (figure 2, interactions non représentées). Les données les plus récentes montrent que la transcriptase inverse du HIV-1 (RTp66/p51) est capable de transcrire l'ARN viral, même très étroitement associé à des protéines $\mathrm{NCp} 7$. Cette transcription inverse est rapide et relativement fidèle in vitro puisque aucune erreur n'a pu être constatée sur plusieurs centaines de nucléotides polymérisés. Tel qu'il est admis, le processus de transcription inverse commence par l'allongement de l'ARNt ${ }^{\text {Lys }, 3}$ jusqu'au début de TAR (figure 3), et la suite nécessite le transfert de ce court fragment d'ADN de sens négatif (appelé en anglais minus strand strong stop DNA) en $3^{\prime}$ du génome. Sur la base de données biochimiques et génétiques, il est apparu que ce transfert de brin d'ADN (l'équivalent d'une recombinaison homologue), nécessitait l'élément TAR en 5' et 3' du génome, l'activité $\mathrm{RNaseH}$ associée à la transcription inverse, ainsi que la protéine NCp7. A la fin de la synthèse du provirus, les protéines NCp7 se trouvent associées à l'ADN double brin et pourraient être impliquées dans la présentation des extrémités de l'ADN à l'intégrase [11].

Des analyses du transfert de brin d'ADN ont révélé que de nombreuses erreurs intervenaient au site même du transfert. Parmi les questions soulevées, on peut en retenir

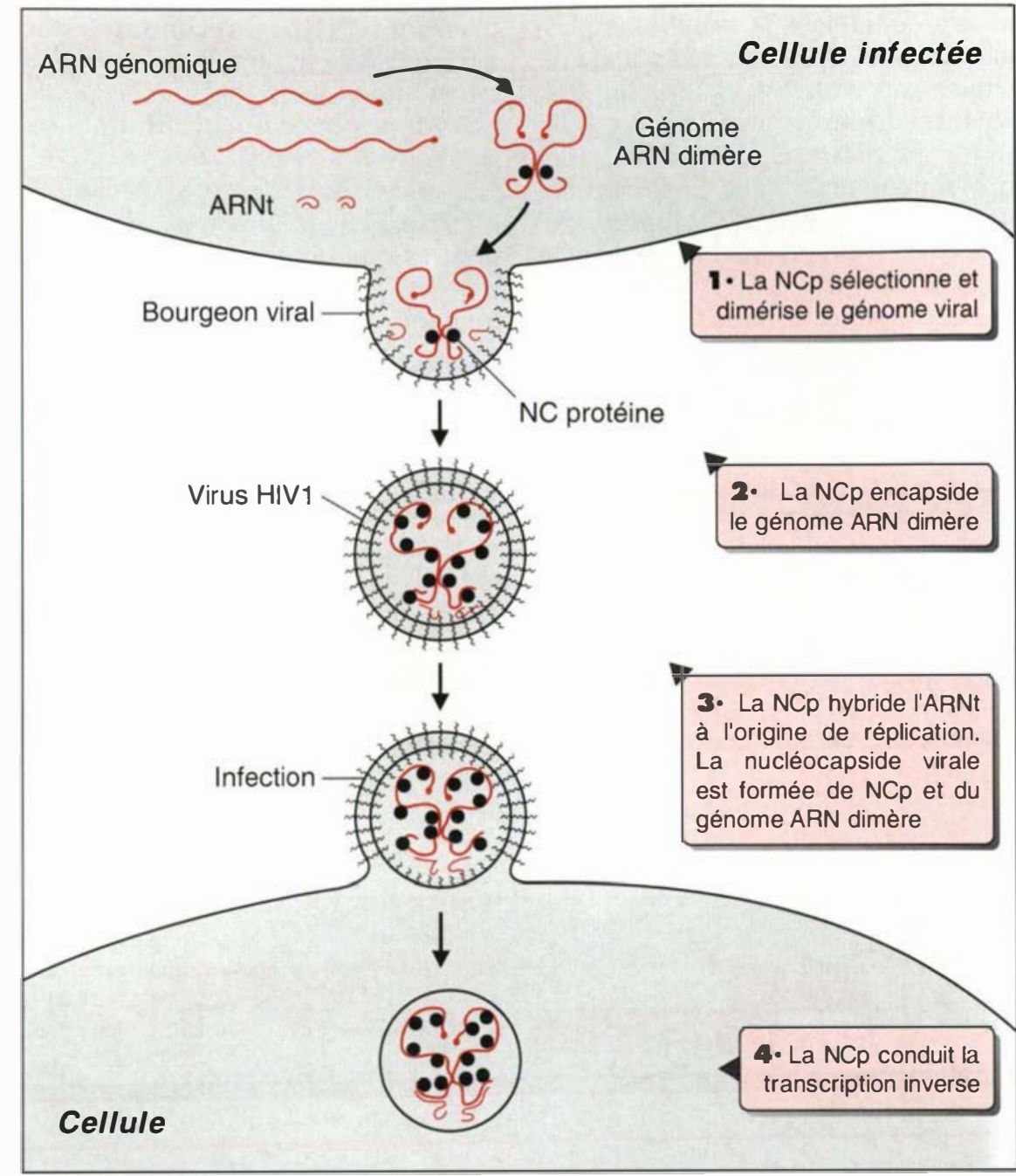

Figure 2. La protéine de nucléocapside NCp 7 au cours du cycle réplicatif du HIV-1. Au stade 1, la protéine NCp7, mûre et/ou sous forme de précurseur Gag, reconnaît I'ARN viral non épissé, ce qui induit la formation d'une ribonucléoprotéine contenant le génome sous forme dimérique len ronds noirs: la protéine $\mathrm{NCp} 7$ ). Au stade 2, le bourgeon viral se forme et entraîne l'encapsidation du génome dimérique, probablement sous forme de nucléoprotéine. La maturation des précurseurs Gag et Gag-Pol se fait de concert avec la formation des bourgeons et la libération des virions par la cellule infectée. Au stade 3, c'est sans doute au cours des derniers événements conduisant à la formation des virions que la NCp 7 induit l'hybridation de l'amorce réplicative ARNLvs, 3 au PBS. A ce stade, un des rôles majeurs de la protéine NCp7 est de protéger le génome de l'action des nucléases extracellulaires, puis intracellulaires, après l'infection. Au stade 4, la transcription inverse se fait au sein de la nucléocapside, et les sauts de la transcriptase inverse pour synthétiser le provirus semblent être guidés par la protéine NCp7. 


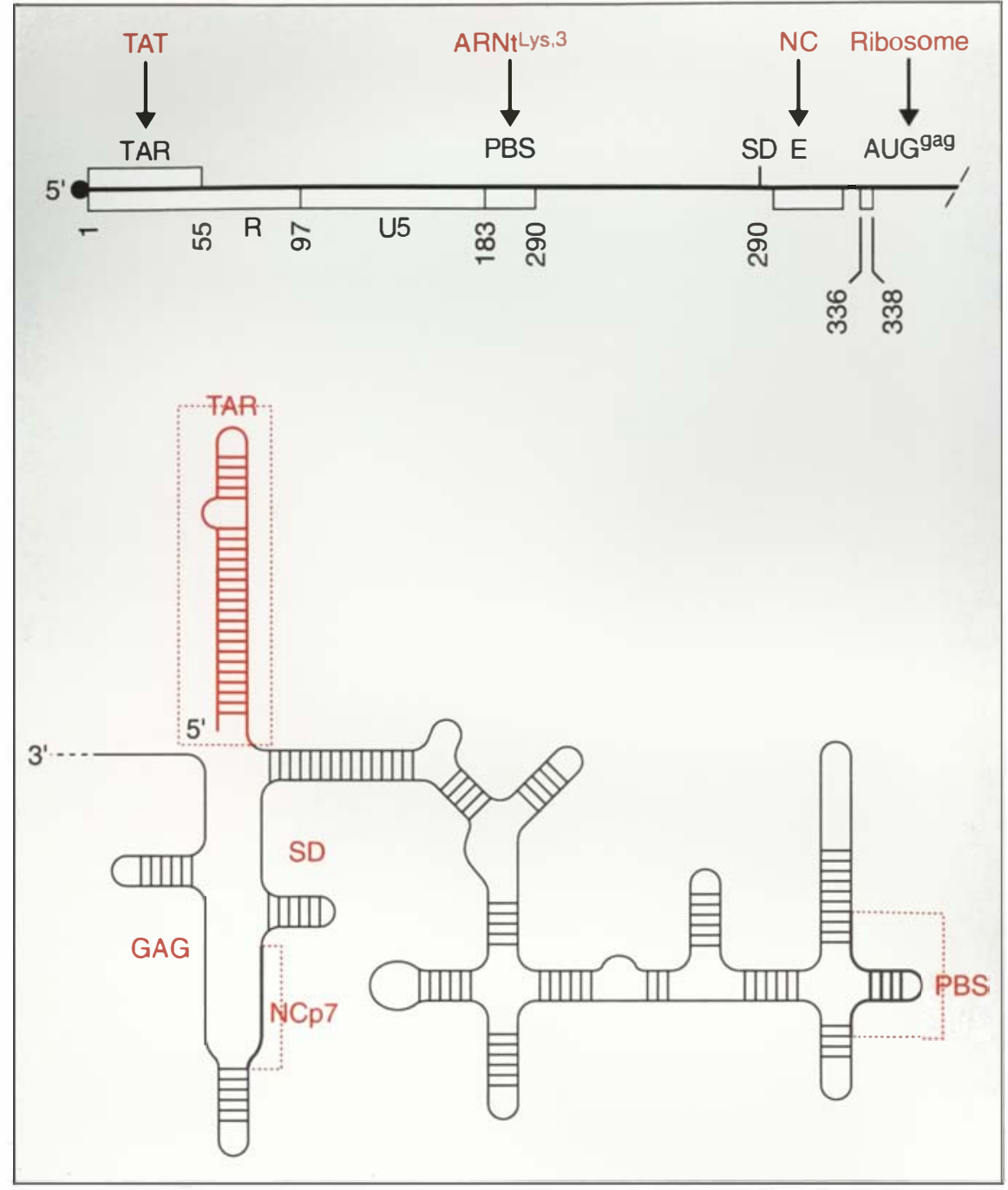

Figure 3. Structure secondaire du leader $5^{\prime}$ du génome ARN du virus HIV-1. La ligne supérieure correspond à la carte génétique des 500 premiers nucléotides de I'ARN génomique HIV-1. Le schéma de structure secondaire, présenté en dessous, reproduit les données obtenues avec I'ARN synthétisé in vitro du HIV-1 mal $[7,8]$. Les différents éléments régulateurs sont, de $5^{\prime}$ en $3^{\prime}$ : TAR (transcription du provirus et saut lors de la transcription inverse) ; PBS (hybridation de I'ARNtLvs, 3 et initiation de la transcription inverse) ; $S D$ (épissage) ; NCp 7 (site de fixation de la protéine $N C p 7$, probablement lié à la dimérisation/encapsidation de l'ARN viral; [6]); GAG (initiation de la traduction). II est à noter que le site reconnu par la NCp7 et le signal de dimérisation:encapsidation (probablement du site $S D$ à un site situé en $3^{\prime}$ de l'AUG de Gag) ne se retrouvent que dans le génome viral non épissé (pré-génome).

$\mathrm{m} / \mathrm{s} n^{\circ} 8.9$ vol. 9, août-seplembre 93 trois: sachant que le génome est dimérique au sein de la nucléocapside, d'autres événements de recombinaison peuvent avoir lieu lors de la synthèse du provirus [12] ; sont-ils accompagnés d'erreurs ? La structure du génome HIV-1 peut-elle être la source de "points chauds " de recombinaison? Quelle peut être la contribution de la protéine $\mathrm{NCp} 7$ à la fréquence des recombinaisons et aux erreurs associées lors de la synthèse du provirus $\mathrm{HIV}-1$ ?

Le fait que les interactions entre le génome ARN dimérique et les molécules de NCp7 soient très stables a aussi été discuté lors de ce colloque. En effet, ces données expliquent dans une certaine mesure pourquoi la nucléocapside du HIV-1 est assez résistante aux protéases et nucléases extra- et intra-cellulaires. Ainsi, des mutations dans la protéine $\mathrm{NCp} 7$ qui déstabilisent les interactions ARN/protéine NCp7 conduisent à une dégradation rapide du génome et à une perte du pouvoir infectant du virus in vivo [13].

Compte tenu du rôle crucial des protéines de nucléocapside dans l'architecture et le pouvoir infectant des rétrovirus, il était important de déterminer la structure tridimensionnelle de la NCp7 du HIV-1 afin de caractériser, au niveau moléculaire, les domaines de la protéine impliqués plus spécifiquement dans certaines de ses fonctions. Outre la possibilité de fournir une base structurale aux mécanismes d'action de la NCp7, ces recherches représentent une nouvelle voie dans l'élaboration d'anti-viraux sélectifs. Du fait de sa taille assez réduite, la $\mathrm{NCp} 7$ a été synthétisée en phase solide en quantités importantes (> $100 \mathrm{mg}$ ) [14], et avec un grand degré de pureté (99\%), permettant d'étudier sa structure en solution par résonance magnétique nucléaire (RMN) à haut champ et fluorescence [15]. De nombreux mutants mutants ponctuels obtenus par remplacement d'un acide aminé ou mutants comportant la délétion d'un ou plusieurs domaines - ont été également préparés par synthèse. Les propriétés de ces peptides dérivés de la NCp7 ont été étudiées in vitro à l'aide d'un fragment d'ARN HIV-1 correspondant au leader 5' (figure 3), 
puis comparées à celles obtenues in vivo en introduisant des mutations semblables dans un clone moléculaire infectieux du HIV-1.

\section{Étude conformationnelle de la pro- téine $\mathrm{NCp} 7$}

Le premier résultat a été de constater que la $\mathrm{NCp} 7$ synthétique était aussi active in vitro que le précurseur NCpl5 (NCp7 + p6) extrait des virions HIV-1 ou produit dans $E$. coli, démontrant que tous les éléments fonctionnels de la protéine, nécessaires aux activités "hybridases" ARN:ARN, se trouvaient dans la NCp7 mûre. La structure tridimensionnelle de la NCp7 a donc été déterminée dans l'eau par RMN du proton à $600 \mathrm{MHz}$ [16]. Cette étude a été facilitée par l'analyse préalable du fragment (13-51) qui comprend les deux doigts à zinc. L'interprétation classique des spectres de RMN à deux dimensions (COSY et NOESY) permet non seulement d'attribuer tous les protons appartenant à un même acide aminé et de déterminer l'enchaînement de ces acides aminés, mais également de mettre en évidence les éventuels rapprochements spatiaux entre protons éloignés dans la séquence peptidique. On obtient ainsi une liste de distances interhydrogènes qui est introduite dans le logiciel de distance-géométrie DIANA [17] afin d'engendrer des structures tridimensionnelles compatibles avec les contraintes expérimentales. Les structures sont ensuite minimisées, ce qui permet d'accéder au modèle représenté dans la figure 4.

Les caractéristiques structurales de la NCp7 peuvent se résumer en trois points essentiels : (1) les deux doigts à zinc sont fortement structurés autour des atomes de zinc. Ceux-ci sont liés, selon une géométrie tétrahédrique, par les atomes de soufre des trois cystéines et l'azote $\epsilon$ de l'histidine ; (2) il existe un rapprochement important entre les deux doigts, mis en évidence par la courte distance entre les acides aminés aromatiques $\mathrm{Phe}^{16} \mathrm{du}$ premier doigt et $\operatorname{Trp}^{37} \mathrm{du}$ second. Cette proximité dépend, entre autres, d'un résidu proline en position 31 qui impose un coude à la courte chaîne peptidique RAPRKKG reliant les deux doigts ;

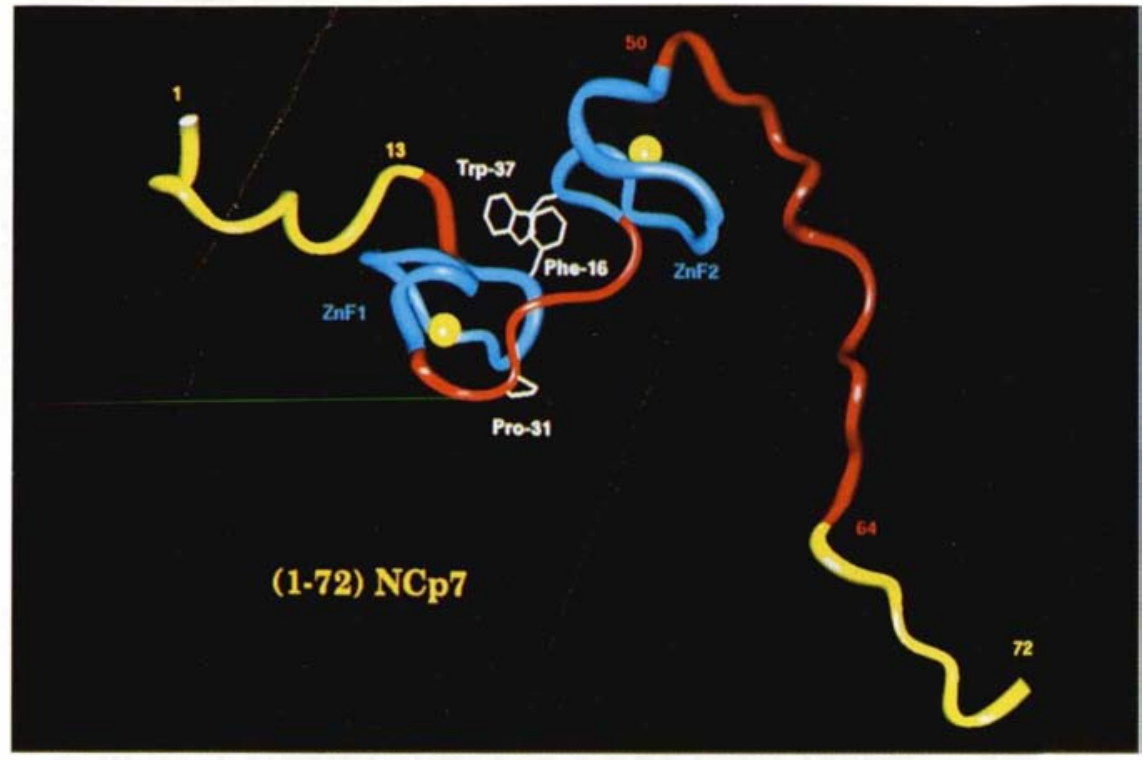

Figure 4. Représentation générale en ruban de la NCp7 du HIV-1. Les domaines $\mathrm{N}$ - et $\mathrm{C}$-terminaux sont indiqués (jaune), les domaines nécessaires à l'activité de la protéine in vitro sont en rouge, et les doigts à zinc sont en bleu (Nathalie Jullian) (voir figure 1 pour la localisation des acides aminés aromatiques Phe-16 et Trp-37.)

(3) les fragments $\mathrm{N}$ - et C- terminaux, de part et d'autre des deux doigts, sont flexibles, mais la région (53-64) comprenant le domaine hydrophobe qui encadre un résidu lysine (Lys ${ }^{59}$ ) isolé, conserve un arrangement spatial relativement bien défini. La signification fonctionnelle de ces éléments structuraux a pu être démontrée grâce à la synthèse et à l'analyse structurale de NCp7 modifiées. Ainsi, le remplacement de $\mathrm{Pro}^{31}$ par l'isomère D.Pro change l'orientation du domaine de liaison et conduit à éloigner les deux doigts. Ce phénomène se traduit par la disparition de l'effet Overhauser* entre les résidus Phe et Trp, qui, dans la NCp7, reflétait leur proximité. Des expériences de transfert de fluorescence d'un résidu Tyr, introduit à la place de Phe dans la

\footnotetext{
* L'effel Overhauser consiste en une modification de l'intensité du signal de résonance d'un noyau, hydrogène par example, lorsqu'il est spatialement proche d'un autre. L'intensité du phénomène est reliée à la distance qui sépare les doux no yaux selon une relation en $1 / r^{6}$. La mesure de cet effel est donc une méthode direcle de mesure de distance par exemple entre les protons d'une molécule, sa limite supérieure étant de l'ordre de $5 \AA$. Les effets "NOE " sont ensuile introduits en terme de contraintes de distances dans des programmes de calcul qui reconstituent la structure de la molécule (modélisation moléculaire).
}

NCp7 et ses mutants, permettent de confirmer ces données RMN (Y. Mély).

La protéine D.Pro ${ }^{31}$ NCp7 semble avoir perdu, dans une large mesure, ses activités in vitro. Il était essentiel de vérifier la nature des perturbations causées in vivo par des changements du résidu Pro ${ }^{31}$. La substitution, par mutagenèse dirigée, d'un résidu Leu à la place de $\mathrm{Pro}^{31}$ dans un clone moléculaire infectieux du HIV-1 conduit à la perte totale du pouvoir infectant du HIV-1 muté (M. Ottmann). L'importance de la géométrie des doigts à zinc, dont l'arrangement du type $\mathrm{CX}_{2} \mathrm{CX}_{4} \mathrm{HX}_{4} \mathrm{C}$ est très conservé, a été mis en évidence par synthèse d'une protéine $\mathrm{NCp} 7$ dans laquelle l'histidine du premier doigt a été remplacée par une cystéine. Le domaine digital $\mathrm{CX}_{2} \mathrm{CX}_{4} \mathrm{CX}_{4} \mathrm{C}$ lie parfaitement le zinc, mais la structure est très modifiée, faisant disparaître, là encore, le rapprochement entre les résidus Phe et Trp. In vitro, on a pu constater que la protéine avait perdu la totalité de ses propriétés biologiques et que, reproduite par mutagenèse dirigée, cette modification entraîne la formation de virus non infectieux (S. Saragosti, M. Ott$\mathrm{m} / \mathrm{s} n^{\circ} 8.9 \mathrm{vol} .9$, août-septembre 93 
mann, Ecole normale supérieure, Lyon, France).

Le rôle des doigts à zinc a été exploré par la synthèse d'une protéine dédigitalisée (appelée NCp7dd) (voir Tableau I, peptide 5) dans laquelle les doigts ont été remplacés par un dipeptide Gly-Gly pour lier les différents domaines [18]. Cette NCp7dd est aussi active que la NCp7 in vitro. On constate, par ailleurs, que le plus petit peptide actif est la NCp7 13-64 avec (peptide 2) ou sans les deux doigts (peptide 6). Il est également intéressant d'observer que la $\mathrm{NCp} 7$ (29-64) (peptide 3) et que la NCp7 (13-64, D.Pro) (peptide 4) ne sont plus capables de dimériser l'ARN viral mais possèdent encore une faible activité leur permettant d'hybrider l'ARNt ${ }^{\text {Lys,3 } 3}$ au monomère. L'absence d'activité in vitro de la NCp7 (13-64), dans laquelle la lysine 59 a été remplacée par une sérine (peptide 8), démontre l'importance des résidus basiques, et plus particulièrement de ce résidu qui est situé dans une séquence hydrophobe (53-64), bien conservée dans les protéines de nucléocapside.

Enfin, les domaines ${ }^{13} \mathrm{VK},{ }^{29} \mathrm{RAPR}$ KKG et ${ }^{55}$ NFLGKIWPSY doivent être liés de façon covalente puisque le mélange de ces fragments ne possède aucune activité in vitro vis-à-vis de l'ARN viral et de l'amorce ARNt ${ }^{\text {Lys,3 }}$. Le rôle des doigts (ou des dipeptides Gly-Gly) est donc de rapprocher dans l'espace ces domaines dans la NCp7 [18]. L'affinité considérable de la $\mathrm{NCp} 7$ pour le zinc $\left(10^{13} \mathrm{M}^{-1}\right)$ [15], déterminée par des expériences de fluorescence, permet de comprendre pourquoi la protéine demeure complexée au zinc dans les virus [19].

Ces résultats conduisent à s'interroger sur le rôle des doigts à zinc in vivo. Des résultats préliminaires (Tableau I), obtenus avec des protéines NCp7 mutées dans lesquelles les mutations entraînent une modification de la structure des doigts (N. Jullian), indiquent que ces derniers seraient nécessaires à la formation d'une capside et d'une nucléocapside stables (M. Ottmann). Comment? Les doigts à zinc contribueraient à rendre coopératives les interactions entre les molécules de $\mathrm{NCp} 7$ et le génome $\mathrm{ARN}$ dimérique in vivo au moyen d'interactions protéiques conduisant à un arrangement ordonné des NCp7 le long de l'ARN (N. Morellet). Ainsi, des mutations modifiant les doigts à zinc entraînent une instabilité du virus et une dégradation rapide du génome par des nucléases ([13], M. Ottmann).

Les études, par RMN et fluorescence, des interactions entre la $\mathrm{NCp} 7$ et un oligonucléotide modèle se poursuivent, dans le but de déterminer quels sont les acides aminés impliqués, et quels changements de structure de la protéine NCp7 et de l'acide nucléique sont induits par leur rencontre. Les études continuent aussi, in vitro et in vivo, au moyen de la biochimie et de la génétique moléculaire, pour déterminer le rôle exact des acides aminés basiques de la NCp7 dans les diverses fonctions de la protéine, et confirmer ainsi les grandes lignes des relations structure:fonctions de la NCp7 dans un contexte viral.

C'est aussi grâce à la disponibilité de plusieurs anticorps monoclonaux, préparés à partir de NCp7 de synthèse, qui reconnaissent soit un épitope $\mathrm{N}$ ou C-terminal, soit la structure tridimensionnelle de la protéine, que les relations structure:fonctions de la NCp7 mûre et dans le précurseur Gag commencent à être analysées in vivo ( $R$. Benarous, Th. Delaunay, V. Tanchou, ICGM, Paris).

L'analyse de la structure dimérique de l'ARN HIV-1 est en cours par l'équipe de B. Ehresmann (IBM du Cnrs, Strasbourg, France); son décryptage promet d'être passionnant car elle semble impliquer des conformations très particulières, dont une quadruple hélice d'ARN. La mise au jour de cette structure dimérique permettra aussi de comprendre comment la protéine $\mathrm{NCp} 7$ chaperonne deux molécules d'un même ARN pour donner un génome rétroviral diploïde.

\section{Conclusion}

La protéine de nucléocapside $\mathrm{NCp} 7$ du HIV-1, à l'image de celle d'autres rétrovirus, à la fois est un élément fondamental de la structure du virus, et est douée d'activités liées à la reconnaissance de l'ARN viral et de l'amorce réplicative $A \mathrm{RNt}^{\mathrm{Lys}, 3}$. Cette plurifonctionnalité de la NCp7 la désigne comme une cible de choix d'agents anti-HIV. Un système de criblage d'agents anti-NCp7 est mis en place par Rhône-Poulenc Rorer af in de sélectionner des agents capables d'inhiber la protéine NCp7 au cours de la morphogenèse du HIV-1 et la synthèse du provirus. En outre, la mise en évidence, dans la NCp7, de domaines discrets, impliqués dans
Tableau I

ACTIVITÉ «ARN HYBRIDASE » DE DIFFÉRENTES FORMES DE NCp7 DU HIV-1

\begin{tabular}{|ll|c|c|}
\hline \multicolumn{1}{|c|}{ Peptide } & Dimérisation & $\begin{array}{c}\text { Hybridisation } \\
\text { de I'ARNt Lys.3 }\end{array}$ \\
\hline 1 & NCp7 & +++ & +++ \\
2 & $(13-64) N C p 7$ & +++ & +++ \\
3 & $(29-64) N C p 7$ & - & + \\
4 & D.Pro (13-64)NCp7 & - & + \\
5 & C23(13-64)NCp7 & - & N. D. \\
6 & NDp7 dd & +++ & +++ \\
7 & $(13-64) N C p 7$ dd & +++ & +++ \\
8 & Ser59(13-64)NCp7 dd & - & N. D. \\
\hline
\end{tabular}

Les différentes formes de NCp7 sont indiquées de 1 (protéine virale intacte) à 8. "Dimérisation " concerne la dimérisation de I'ARN HIV-1 des positions 1 à 415 (voir figure 3 ), et "hybridation de l'ARNt'ys, 3 " le positionnement de l'amorce réplicative ARNt au PBS (voir figure 3). Les signes,+++ et +++ indiquent une activité égale à 20-30\%, $50-60 \%$ et $80-100 \%$ de l'activité optimale [6-18]. N.D. : non déterminé. 
la morphogenèse du virus HIV-1 et sa réplication, permet d'envisager le développement de leurres peptidomimétiques (M.-C. Fournié-Zaluski, C.Z. Dong, faculté de pharmacie, Inserm U. 266, Paris, France).

Le prochain colloque sur la nucléocapside du HIV-1 se tiendra dans un an et nul doute que beaucoup de nouveaux résultats y seront présentés, enrichissant encore nos connaissances sur les fonctions de la NCp7, la structure dimérique du génome diploïde du HIV-1, et la façon de les inhiber

Jean-Luc Darlix

Ecole normale supérieure de Lyon (ENSL), Laboratoire Rétro Inserm, 46, allée d'ltalie, 69364 Lyon Cedex 07, France.

Bernard-Pierre Roques

Faculté de pharmacie, Inserm U. 266, 4, avenue de l'Observatoire, 75006 Paris, France.

\section{RÉFÉRENCES}

1. Darlix JL. Éléments de structure et de variabilité du rétrovirus humain HIV-1. Bull Inst Pasteur 1991; 89 : 211-42.

2. Tounekti N, Mougel M, Roy C, Marquet R, Darlix JL, Paolctti J, Ehresmann B Ehresmann C. Effect of dimerization on the conformation of the encapsidation Psi domain of Moloney murine leukemia virus RNA. J Mol Biol 1992 ; 223 ; 205-20.

3. Housset $\mathrm{V}$, de Rocquigny $\mathrm{H}$, Roques $\mathrm{B}$, Darlix JL. Basic amino acids flanking the zinc linger of Moloney murine leukemia virus NCp1O protein are critical for virus infectivity. J Virol 1993 ; 67 : 2537-49.

4. Darlix JL, Gabus C, Allain B. Analytical study of avian reticuloendotheliosis virus RNA generated in vivo and in vitro. J Viro $1992 ; 66: 7245-52$

5. De Rocquigny $\mathrm{H}$, Ficheux D, Gabus C, Allain B, Fournié-Zaluski MC, Darlix JL, Roques B. Two short basic sequences surrounding the zinc finger of $\mathrm{NCp} 1 \mathrm{O}$ protein of Moloney murine leukemia virus are critical for RNA annealing activity. Nucleic Acids Res 1993 ; 21 : 823-9.

6. Darlix JL, Gabus C, Nugeyre MT, Clavel F, Barré-Sinoussi F. Cis elements and trans acting factors involved in the RNA dimerization of HIV-1. J Mol Biol 1990 ; $216: 689-99$

7. Baudin F, Marquet R, Isel C, Darlix JL Ehresmann B, Ehresmann C. Functiona sites in the 5' region of $\mathrm{HIV}-1$ RNA form defined structural domains. J Mol Biol 1993 ; 229 : 382-97

8. Marquet R, Baudin F, Gabus C, Dar lix JL, Mougel M, Ehresmann C, Ehresmann B. Dimerization of HIV-1 RNA : stimulation by cations and possible mecha nism. Nucleic Acids Res 1991 ; 19 : 2349-57.

9. Hong S, Boulanger P. Assembly defective point mutants of HIV-1 GAG precursor phenotypically expressed in recombinan baculovirus infected cells. J Virol 1993; 67 2787-98

10. Smith A Srinivasakumar N, Ham marskjold ML, Rekosh D. Requirements for incorporation of Prl60gag-pol from HIV-1 into virus particles. J Virol 1993; 67 2266-75.

11. Lapadat-Tapolsky $M$, de Rocquigny $H$ van Gent D, Roques B, Plasterk R, Dar- lix JL. Interactions between HIV-1 nucleocapsid protein and viral DNA may have important functions in the viral life cycle. Nucleic Acids Res 1993; 21 : 831-39.

12. $\mathrm{Hu} \mathbf{W}, \mathrm{Temin} \mathrm{H}$. Genetic consequence of packaging two RNA genomes in one retroviral particle : pseudodiploidy and high rate of genetic recombination. Proc Natl Acad Sci USA 1990 ; 87 : 1556-60

13. Aronoff R, Hajjar A, Linial M. Avian retroviral encapsidation : reexamination of functional 5' RNA sequences and the role of nucleocapsid Cys-His motifs. J Virol $1993 ; 67$ : 178-88.

14. De Rocquigny $\mathrm{H}$, Ficheux D, Gabus $\mathrm{C}$ Fournié-Zaluski MC, Darlix JL, Roques B. First large scale chemical synthesis of the 72 amino acid HIV-1 nucleocapsid protein NCp7 in an active form. Biochem Biophys Res Commun 1991; $180: 1010-8$.

15. Mély $\mathrm{H}$, de Rocquigny $\mathrm{H}$, Piémont $\mathrm{E}$ Deméné H, Jullian N, Fournié-Zaluski MC, Roques B, Gérard D. Influence of the $\mathrm{N}$ and $\mathrm{C}$-terminal chains on the zinc binding and conformational properties of the central zinc finger structure of MoMuLV nucleocapsid protein : a steady state and time resolved fluorescence study. Biochem Biophys Acta 1993 ; 1161 : 6-18.

16. Morellet $\mathbf{N}$, Jullian $\mathbf{N}$, de Rocquigny $\mathrm{H}$, Maigret B, Darlix JL, Roques B. ${ }^{1} \mathrm{H}$ NMR derived conformation of the nucleocapsid protein $\mathrm{NCp} 7$ of human immunodeficiency virus type 1.EMBO / 1992 ; Il : 3059-65.

17. Guntert P, Braun W, Wuthrich K. Efficient computation of three dimensional protein structures in solution from nuclear magnetic resonance data using the program DIANA and the supporting programs CALIBA, HABAS and GLOMSA. I Mol Biol 1991 ; 217 : 517-530.

18. De Rocquigny $\mathrm{H}$, Gabus $\mathrm{C}$, Vincent $\mathrm{A}$, Fournié-Zaluski MC, Roques B, Darlix JL. Viral RNA annealing activities of HIV-l nucleocapsid protein require only peptide domains outside the zinc fingers. Proc Natl Acad Sci USA 1992 ; 89 : 6472-6.

19. Summers M, Henderson L, Chance M et al. Nucleocapsid zinc fingers detected in retroviruses : EXAFS studies of intact viruses and the solution-state structure of the nucleocapsid protein from HIV-1. Prot Science $1992 ; 1: 563-74$
TIRÉS A PART

J.-L. Darlix.
Cette mini-synthèse est le compte rendu du colloque "Basic Features of the Nucleocapsid of the Human Immunodeficiency Virus Type 1 ", Lyon, 5-6 avril 1993 et qui a été soutenu par I'ENS de Lyon et Synthelabo-Recherches. Les travaux sur la nucléocapside HIV-1 sont effectués dans le cadre de l'Action Coordonnée 14 de I'Agence Nationale de Recherches sur le SIDA (ANRS, Directeur Jean-Paul Lévy). 